\title{
Points Review of Kawasaki as an Interesting Disease
}

\author{
Abdollah Karimi ${ }^{1, *}$ \\ ${ }^{1}$ Pediatric Infections Research Center, Shahid Beheshti University of Medical Sciences, Tehran, IR Iran \\ ${ }^{*}$ Corresponding author: Abdollah Karimi, Pediatric Infections Research Center, Shahid Beheshti University of Medical Sciences, Tehran, IR Iran. Tel/Fax: +98-2122226941, E-mail: dr \\ akarimi@yahoo.com.
}

Received: Jun 11, 2013; Accepted: Jun 22, 2013

Keywords: Mucocutaneous Lymph Node Syndrome

Mucocutaneous lymph node syndrome (MCLN) or Kawasaki disease (KD) is a notable disease of unknown etiology and although pediatricians are familiar with different aspects of it, they tend to make under and over diagnosis which can result in cardiovascular disorders, misapply of intravenous immunoglobulin (IVIG) and its consequences which can also cause a delay in diagnosis of mimicking diseases (1-3).

Therefore, the following recommendations must be taken seriously:

1. To diagnose KD putting aside other causes of similar symptoms is needed, and since there are, extensive various diseases that are mistaken for it, we need history, precise clinical examination, strong reasoning and clinical judgment to diagnose well-timed promptly. Thus, to achieve this, efficient knowledge, long-term experience and patience are of high importance as negligence will result in mismanagement $(2,4)$.

2. Due to this fact that KD can be related with some infections by complicated mechanisms, thus, if some bacterial infections which should be improved in the expected-time with a delay of some days, goes into a new phase, and reveals KD manifestations, we must consider KD as complication of infection and manage it properly (2).

3. We should take it into consideration that KD doesn't have any particular test, so diagnosis, must depend on definite criteria and valid clinical assay otherwise other differential diagnosis can be mistaken for it sometimes or we may neglect coronary artery (COA) involvements and aneurysm and in some cases sudden death may happen in sub-acute stage $(5,6)$.

4. It is recommended to use clinical laboratory manifestations and echocardiographic features to diagnose KD especially in incomplete disease; however clinical signs and symptoms have the most important role (7).
5. In cases of doubt over KD diagnosis, consultation of the high-level expert will help us avoid dangerous complications, wandering of patient and undiagnosed illnesses of differential diagnosis (7).

6. During 1-2 weeks after diagnosed and treated or missed KD, deep transverse grooves on fingernails (Beau's Sign) may be seen, however then echocardiography will be needed, it isn't pathognomonic for KD and other differential diagnosis also should be remarked $(1,8,9)$.

7. Since KD is a systemic disease, paying attention to organ involvement such as CNS, lung, kidney, liver and digestive system in incomplete cases beside hematocrit (Hct), albumin (Alb), transaminase (TA) and urine analysis (U/A) assay remarkably help us to diagnose and rule out KD or its differential diagnosis (1).

8. In less than $2 \%$ of KD cases, specially, in complicated cases of KD and macrophage activated syndrome, thrombocytopenia will be found instead of thrombocytosis, thus low level of platelets whereas clinical signs and symptoms are in accordance with diagnostic criteria doesn't rule out KD existence, so great caution is needed (7).

9. It would be better to rely on clinic, scientific reasoning, right judgment and plain inexpensive tests instead of costly experiments such as N-terminal pro-B-type natriuretic peptide (NT-ProBNP) (revealed diastolic dysfunction) which are not available everywhere $(10,11)$.

10. Abnormality and aneurysm of medium artery especially coronary artery is the hallmark of disease, but since it has been seen in $25 \%$ of untreated cases, lack of COA involvement doesn't rule out it. On the other hand diagnosis of COA involvements depends on individual experience; therefore unclear echocardiographic reports should be approached cautiously in order to avoid making mistakes about KD (2).

11. Coronary arteries are small in infants, thus, paying 
attention to patients age for labeling dilated coronary arteries in them is very important $(3,12)$.

12. In the event that COA involvement doesn't happen and acute phase reactant (APR) and platelets return to normal during 8 weeks after acute stage, it will not happen next time. In these circumstances, frequent echocardiography test order yearlong will be waste of time, energy and expenses $(1,7)$.

13. Regarding to KD, echocardiography should be ordered for all febrile infants without local signs and symptoms for more than one week whom their erythrocyte sedimentation rate (ESR) and C-reactive protein (CRP) levels are respectively higher than $40 \mathrm{~mm} / \mathrm{h}$ and $3 \mathrm{mg} / \mathrm{dL}$; even if there are no sign and symptoms related to $\operatorname{KD}(1,3,7)$.

14. There is a wide-spectrum of diseases which should be considered before labeling of KD such as scarlet fever, measles, sepsis, inflammatory disorders (like juvenile idiopathic arthritis (JIA) and polyarteritis nodosa (PAN)), diseases with fever and rash and malignancies which share the same symptoms as $\mathrm{KD}$; this makes the awareness of physicians in encountering the similar diseases to KD extremely necessary $(3,11)$.

15. In any extraordinary febrile disease such as idiopathic gangrene in extremities, renal and lung involvements, atypical KD should be taken considerable as one of significant differential diagnosis even if it doesn't fulfill KD criteria (1).

16. Presence of some symptoms such as purulent conjunctivitis, vesicular rash, and exudative pharyngitis (although rarely can be seen in KD) makes the diagnosis of Kawasaki disease less likely (13).

17. In failure of KD treatment with no response to proper therapy, we should revise our management and also be aware of other diseases like PAN, JIA, lymphoma etc. (2).

18. Due to the point that some collagen vascular diseases (CVD) such as JIA and PAN are significant differential diagnosis of $\mathrm{KD}$, in suspicious cases particularly incomplete ones, tests related with JIA and PAN before IVIG therapy should be requested, because IVIG can cause significant interference in any test such as rheumatic factor (RF) or Anti-nuclear antibody ANA and may cause delay in diagnosis of above mentioned diseases by creating both false positive and false negative results (14).

19. Incomplete KD doesn't imply mildness state of it and risk of COA involvement manifestation will not be less than the classic disease, there is no difference in following up them $(3,7,12,15)$.

20. Although high level of ESR and platelets is an ordinary laboratory finding in KD patient, there are different diseases which may increase these tests such as infectious, inflammatory disorders and malignancies, thus in absence of KD criteria either, classic or incomplete, we shouldn't make parents worry, and beside right assay we should not prescribe IVIG on the base of above mentioned tests. Applying wrong policy will cause delay in diagnose and waste of time, energy and hurt the patients (7).

21. It should be mentioned that during 3 months after
KD occurrence, vaccines even inactivated vaccines may trigger a relapse, thus if there isn't an urgency to prescribe, some experts recommended to postpone injection to 3 months after disease, about measles and varicella vaccines, it must be prescribed 11 months after IVIG therapy to achieve the best immune response (1).

22. In children affected by Kawasaki, paying attention to influenza symptoms is necessary-especially in flu seasons and Aspirin prescribing should be avoided because of Reye's syndrome incidence risk (16).

\section{Authors' Contribution}

There is only one author for this study.

\section{Financial Disclosure}

There is no conflict of interest.

\section{References}

1. Dajani AS, Taubert KA, Gerber MA, Shulman ST, Ferrieri P, Freed M, et al. Diagnosis and therapy of Kawasaki disease in children. Circulation. 1993;87(5):1776-80.

2. Newburger JW, Takahashi M, Gerber MA, Gewitz MH, Tani LY, Burns JC, et al. Diagnosis, treatment, and long-term management of Kawasaki disease: a statement for health professionals from the Committee on Rheumatic Fever, Endocarditis, and Kawasaki Disease, Council on Cardiovascular Disease in the Young, American Heart Association. Pediatrics. 2004;114(6):1708-33.

3. Pickering LK, Baker CJ, Kimberlin DW, Long SS. Red book:2012 report of the committee on infectious diseases. 29th ed. Washington: American academy of pediatrics; 2012. p. 454-460.

4. Scheinfeld N, Steele R. Kawasaki Disease Differential Diagnoses. MED Scape refrences. 2013 [Updated 2013 Feb 15]; Available from: http://emedicine.medscape.com/article/965367-differential.

5. Scheinfeld N, Steele R. Kawasaki Disease Workup. MED Scape refrences. 2013 [Updated 2013 Feb 15]; Available from: http://emedicine.medscape.com/article/965367-workup.

6. Scheinfeld N, Steele R. Kawasaki Disease Treatment and Management.MED Scape refrences. 2013 [Updated 2013 Feb 15]; Available from: http://emedicine.medscape.com/article/965367-treatment.

7. Feigin RD. Feigin and Cherry's textbook of pediatric infectious diseases. Philadelphia: Saunders; 2004.

8. Singh G. Nails in systemic disease. Indian J Dermatol Venereol Leprol. 2011;77(6):646-51.

9. Lopez Neyra A, Alvarez-Coca Gonzalez J, Perez Suarez E, Martinez Perez J, Rubio Villanueva JL. [Beau's lines and Kawasaki disease]. An Pediatr (Barc). 2007;67(6):610-1

10. Alonso-Gonzalez R, Li W. Diastolic dysfunction in patients with Kawasaki disease. Int J Cardiol. 2010;145(3):520-1.

11. Selamet Tierney ES, Newburger JW, Graham D, Baker A, Fulton DR, Colan SD. Diastolic function in children with Kawasaki disease. Int J Cardiol. 2011;148(3):309-12.

12. Crystal MA, Manlhiot C, Yeung RS, Smallhorn JF, McCrindle BW. Coronary artery dilation after Kawasaki disease for children within the normal range. Int J Cardiol. 2009;136(1):27-32.

13. Eidelman Arthur, Feldman-Winter Lori. From the American Academy of Pediatrics: Policy Statement: Breastfeeding and the Use of Human Milk. Pediatrics. 2005;115(2):496-506.

14. Abbott Jordan Keith, Church Joseph A. In vivo assessment of clinically relevant autoantibodies in intravenous immunoglobulin preparations. Pediatr Allergy Immunol Pulmonol. 2010;23(2):121-123.

15. Dodi I, Raggi V, Verna M, Tchana B, Vignali D, Bandello MA, et al. Atipical Kawasaki disease with coronary aneurysm in infant. Ital JPediatr. 2011;37:19.

16. Cox Janelle R, Sallis Robert E. Recognition of Kawasaki disease. Permanente J. 2009;13(1):57. 